\title{
Constraints on wave drag parameterization schemes for simulating the quasi-biennial oscillation. Part II: combined effects of gravity waves and equatorial planetary waves
}

Article

Published Version

Campbell, L. J. and Shepherd, T. G. (2005) Constraints on wave drag parameterization schemes for simulating the quasibiennial oscillation. Part II: combined effects of gravity waves and equatorial planetary waves. Journal of the Atmospheric Sciences, 62 (12). pp. 4196-4205. ISSN 1520-0469 doi: https://doi.org/10.1175/JAS3617.1 Available at https://centaur.reading.ac.uk/32102/

It is advisable to refer to the publisher's version if you intend to cite from the work. See Guidance on citing.

To link to this article DOI: http://dx.doi.org/10.1175/JAS3617.1

Publisher: American Meteorological Society

All outputs in CentAUR are protected by Intellectual Property Rights law, including copyright law. Copyright and IPR is retained by the creators or other copyright holders. Terms and conditions for use of this material are defined in the End User Agreement. 


\section{www.reading.ac.uk/centaur}

\section{CentAUR}

Central Archive at the University of Reading

Reading's research outputs online 


\title{
Constraints on Wave Drag Parameterization Schemes for Simulating the Quasi-Biennial Oscillation. Part II: Combined Effects of Gravity Waves and Equatorial Planetary Waves
}

\author{
Lucy J. CAmpbell* and Theodore G. ShePherd \\ Department of Physics, University of Toronto, Toronto, Ontario, Canada
}

(Manuscript received 28 April 2004, in final form 14 June 2005)

\begin{abstract}
This study examines the effect of combining equatorial planetary wave drag and gravity wave drag in a one-dimensional zonal mean model of the quasi-biennial oscillation (QBO). Several different combinations of planetary wave and gravity wave drag schemes are considered in the investigations, with the aim being to assess which aspects of the different schemes affect the nature of the modeled QBO. Results show that it is possible to generate a realistic-looking QBO with various combinations of drag from the two types of waves, but there are some constraints on the wave input spectra and amplitudes. For example, if the phase speeds of the gravity waves in the input spectrum are large relative to those of the equatorial planetary waves, critical level absorption of the equatorial planetary waves may occur. The resulting mean-wind oscillation, in that case, is driven almost exclusively by the gravity wave drag, with only a small contribution from the planetary waves at low levels. With an appropriate choice of wave input parameters, it is possible to obtain a QBO with a realistic period and to which both types of waves contribute. This is the regime in which the terrestrial QBO appears to reside. There may also be constraints on the initial strength of the wind shear, and these are similar to the constraints that apply when gravity wave drag is used without any planetary wave drag.

In recent years, it has been observed that, in order to simulate the QBO accurately, general circulation models require parameterized gravity wave drag, in addition to the drag from resolved planetary-scale waves, and that even if the planetary wave amplitudes are incorrect, the gravity wave drag can be adjusted to compensate. This study provides a basis for knowing that such a compensation is possible.
\end{abstract}

\section{Introduction}

The quasi-biennial oscillation $(\mathrm{QBO})$ is an oscillation between easterly and westerly zonal winds that is observed in the equatorial stratosphere. It is known to result from momentum transfer via upward-propagating atmospheric waves. Atmospheric general circulation models (GCMs) are able to resolve the planetary-scale equatorial waves (Kelvin waves and mixed Rossby-gravity waves), and the role that these waves play in driving the QBO is well known. In general,

\footnotetext{
* Current affiliation: School of Mathematics and Statistics, Carleton University, Ottawa, Ontario, Canada.
}

Corresponding author address: Dr. T. G. Shepherd, Dept. of Physics, University of Toronto, Toronto, ON M5S 1A7, Canada. E-mail: tgs@atmosp.physics.utoronto.ca
GCMs have nevertheless been unable to generate QBOs with the resolved waves alone and it is now recognized that to generate a large enough forcing to drive the QBO, gravity wave momentum transfer must be included as well (Dunkerton 1997). However, gravity waves, because of their relatively small scale, are in general unresolved in GCMs and their effects must be represented by means of parameterizations. With the addition of parameterized gravity wave drag, GCMs have been able to simulate the QBO (Scaife et al. 2000; Giorgetta et al. 2002; McLandress 2002).

Simulations of the QBO using one-dimensional models have shed some light on the mechanism by which wave-mean flow interactions can generate an oscillation in the mean flow. Holton and Lindzen (1972, hereafter HL72) derived an approximate expression for the drag resulting from thermal damping of equatorial planetary waves, and Plumb (1977) explained the theory in more detail. In Campbell and Shepherd (2005, 
hereafter Part I), we discussed the means by which a QBO-like oscillation can be generated in a onedimensional model using parameterized gravity wave drag alone, and we derived constraints on the relevant input parameters. This is of theoretical interest, since equatorial wind oscillations are generic in planetary atmospheres. However, it is unreasonable to appeal to gravity wave drag as the sole mechanism for driving the terrestrial QBO. With only gravity wave drag, the only way to generate a mean wind oscillation from a zero initial wind state is if the gravity waves are allowed to break without critical levels; for example, the parameter settings discussed in Part I allow the waves to break gravitationally. This is unrealistic for the terrestrial stratosphere where gravity wave breaking is mostly due to critical level interactions, but it is a necessary requirement in a model such as that in Part I that does not include equatorial planetary waves. In the real atmosphere, equatorial planetary waves are clearly an important part of the picture, and thus their effects need to be represented in our model if we wish to gain an understanding of the mechanisms that generate the QBO.

There is a great deal of uncertainty regarding the relative contributions of the two types of waves. In GCMs, the amplitudes of the resolved equatorial planetary waves depend to a great extent on the details of the convective parameterizations used in the models (Horinouchi et al. 2003), and thus there is a lot of variation in planetary wave amplitudes among the different models. In general, even when the planetary wave amplitudes are realistic, additional drag from gravity waves is needed to simulate the QBO accurately. Gravity wave drag generally acts in the direction of the wind in an accelerative sense in the lower parts of the atmosphere, that is, at the levels where the waves first break, and thus tends to amplify wind anomalies. Thus, it can be expected that adding parameterized gravity wave drag to a model that already includes equatorial planetary waves will reinforce a QBO if one existed already, or make it possible to obtain one if the planetary wave forcing was insufficient. Indeed, GCMs have been able to simulate the QBO with different combinations of equatorial planetary waves and gravity waves. Thus, it is important to understand how the two types of wave forcings interact, and in this paper, we seek to understand these interactions. To do this, we add parameterized drag from both types of waves to the onedimensional model that was considered in Part I.

The model comprises the single equation

$$
\frac{\partial \bar{u}}{\partial t}-\nu \frac{\partial^{2} \bar{u}}{\partial z^{2}}=X_{\mathrm{PW}}+X_{\mathrm{GW}},
$$

where $\bar{u}(z, t)$ is the zonally averaged zonal velocity, $v$ is the vertical diffusivity, and $X_{\mathrm{PW}}(z, t)$ and $X_{\mathrm{GW}}(z, t)$ represent the forcing due to equatorial planetary waves and gravity waves, respectively. The equation is solved numerically in a domain extending from a height of 15 $\mathrm{km}$ (around the equatorial tropopause) to a height of $100 \mathrm{~km}$, and the source level of the waves is at the lower boundary. The equatorial planetary wave drag is represented according to the parameterization of HL72. The equatorial planetary waves consist of a westerly Kelvin wave and an easterly anti-Kelvin wave, in which the effect of rotation is ignored, giving a wave with the same characteristics as a Kelvin wave but with a phase speed in the opposite direction. The Kelvin wave/antiKelvin wave combination gives a simple configuration that may be studied to provide insight into the effects of equatorial planetary wave drag. The gravity wave drag schemes used in our investigation are those of Lindzen (1981) and Alexander and Dunkerton (1999, hereafter AD99), both of which are based on the theory of gravity wave breaking and saturation.

We discuss the constraints for generating a QBO-like oscillation with various combinations of the two types of waves and compare these with the constraints derived in Part I for the gravity wave drag schemes, as well as with the constraints on the HL72 scheme when it is used in the absence of gravity wave drag (Plumb 1977). By QBO-like oscillation, we mean an oscillation between easterly and westerly zonal mean winds taking place over a range of heights from the waves' source level up to a height of at least $50 \mathrm{~km}$, with a period of around 700-900 days and with a maximum amplitude within the range of velocities $20-50 \mathrm{~m} \mathrm{~s}^{-1}$.

\section{Equatorial planetary wave drag plus AD99 gravity wave drag}

In this section, we examine the effect of combining the HL72 equatorial planetary wave drag with gravity wave drag, parameterized according to the scheme of Alexander and Dunkerton (1999). First, we recall that, in the absence of gravity waves, the forcing due to a Kelvin wave/anti-Kelvin wave is (Holton and Lindzen 1972; Plumb 1977)

$$
\begin{aligned}
X^{ \pm}(z, t)= & \frac{N \mu e^{-z_{0} / H} F_{0}^{ \pm}}{k^{+}\left[\bar{u}(z, t)-c^{ \pm}\right]^{2} \bar{\rho}_{0}} \\
& \times \exp \left\{-\int_{z_{0}}^{z} \frac{N \mu}{k^{+}\left[\bar{u}\left(z^{\prime}, t\right)-c^{ \pm}\right]^{2}} d z^{\prime}+\frac{z}{H}\right\},
\end{aligned}
$$


where $F_{0}^{ \pm}, c^{ \pm}$, and $k^{ \pm}$are the momentum fluxes of the waves at the source level, their phase speeds, and their horizontal wavenumbers, respectively. In each case, the plus sign denotes the westerly wave and the minus sign the easterly wave. The source level of the waves is $z=$ $z_{0}=15 \mathrm{~km}, H=7 \mathrm{~km}$ is the density scale height, $N=$ $0.02 \mathrm{~s}^{-1}$ is the Brunt-Väisälä frequency, and $\mu$ is the Newtonian cooling rate. The background density is given by $\bar{\rho}(z)=\bar{\rho}_{0} \exp \left[-\left(z-z_{0}\right) / H\right]$, where $\bar{\rho}_{0}$ is the density at the source level. In Part I, we set

$$
\begin{aligned}
F_{0}\left(c^{ \pm}\right) / \bar{\rho}_{0} & =F_{0}^{ \pm} / \bar{\rho}_{0}= \pm 7 \times 10^{-3} \mathrm{~m}^{2} \mathrm{~s}^{-2}, \\
c^{ \pm} & = \pm c_{\mathrm{PW}}= \pm 25 \mathrm{~m} \mathrm{~s}^{-1}, \\
k^{ \pm} & =2 \pi /\left(4 \times 10^{7} \mathrm{~m}\right) .
\end{aligned}
$$

The choice of cooling rate determines the range of heights over which the oscillation takes place. With $\mu=$ $k c^{2} / N H$, there is no exponential increase or decrease in the wave drag with height and the range of QBO altitudes is from the source level up to the top of the computational domain. In the first set of simulations described in this section, we set the cooling rate to be larger than $k c^{2} / N H$ by a factor of 1.5 [with the wave parameters (2.2), this means that $\mu \approx 10^{-6} \mathrm{~s}^{-1}$ ], so that the drag, and hence the amplitude of the oscillation, decreases exponentially with height.

The other important input parameter for the simulation is the vertical diffusivity $\nu$. The choice of $\nu$ affects the form of the oscillation (as discussed in Part I): decreasing $\nu$ increases the period of the oscillation, and in the limit as $\nu \rightarrow 0$, the period becomes infinite, that is, a steady state is attained. When $\nu$ is set to $0.3 \mathrm{~m}^{2} \mathrm{~s}^{-1}$ with the abovementioned combination of input parameters, we obtain a QBO-like oscillation with a period of about 840 days (Fig. 1 in Part I). The maximum QBO amplitude is between 15 and $20 \mathrm{~m} \mathrm{~s}^{-1}$ and occurs between the altitudes 20 and $25 \mathrm{~km}$. The wind goes to zero at the top of the computational domain because of the exponential decrease in the drag.

Let us now examine the effect of adding gravity wave drag to the Kelvin wave/anti-Kelvin wave combination given by (2.2). In the AD99 parameterization, one specifies a spectrum of gravity waves over a range of phase speeds; the waves propagate upward, their amplitudes increasing with height, and each wave breaks and deposits all its momentum at the level where it first becomes statically unstable. A drag profile that is a continuous, or at least piecewise continuous, function of height is obtained when the drag from all the waves in the spectrum is summed up.

We first consider a simple special configuration of the AD99 parameterization that was studied in Part I.
The momentum flux of the waves at the source level is specified as

$$
\begin{aligned}
F_{0}(c) / \bar{\rho}_{0} & =(\operatorname{sgn} c) \times 5 \times 10^{-3} \mathrm{~m}^{2} \mathrm{~s}^{-2} \text { for } \\
-60 & \leq c \leq 60=c_{\max } .
\end{aligned}
$$

With this choice of spectrum, there is an explicit relationship between the phase speeds of the waves $c$ and their breaking levels $z_{b}$, that is,

$$
c^{ \pm}\left(z_{b}\right)=\bar{u}\left(z_{b}\right) \pm \alpha e^{z_{b} / 3 H},
$$

where

$$
\alpha=\left|\frac{2 N}{k \bar{\rho}_{0}} e^{-z_{0} / H} F_{0}(c)\right|^{1 / 3} .
$$

The gravity waves are assumed to have a single horizontal wavenumber; as in Part I, this is chosen to be $k^{ \pm}=2 \pi /\left(4 \times 10^{7} \mathrm{~m}\right)$, the same value used for the planetary waves. As noted in Part I, this is an unrealistically long wavelength for a gravity wave, but since the wave drag is multiplied by an arbitrarily chosen intermittency factor, exactly the same results could be obtained with a more realistic (shorter) gravity wavelength simply by adjusting the values of $F_{0}$ and the intermittency factor $\varepsilon$ in such a way that $F_{0} / k$ and $\varepsilon k$ remain fixed. The phase speed interval $-60 \mathrm{~m} \mathrm{~s}^{-1} \leq c$ $\leq 60 \mathrm{~m} \mathrm{~s}^{-1}$ is discretized into $N_{c}=120$ points, and as in Part I we set the intermittency factor $\varepsilon$ to $2 / N_{c}$. This choice of $\varepsilon$ is consistent with the definition used by AD99 [their (19)]; $\varepsilon$ is proportional to the phase speed resolution and so the calculated drag is unaffected by changes in the resolution. This also means that the total gravity wave input momentum flux of each sign, integrated over the phase speed spectrum, is equal to the input momentum flux of the planetary wave of the same sign. Since the gravity wave and planetary wave spectra are both antisymmetric, the input momentum flux over the whole spectrum sums to zero in each case.

With this choice of input parameters, the gravity wave drag is zero above a level of about $52 \mathrm{~km}$, because by that height all the waves have already broken and deposited their momentum at lower levels. Thus, the possible range of QBO altitudes is from the source level to the 52-km level. As in Part I, the zonal wind at time zero is chosen to be a westerly jet with a maximum of $\bar{u}_{\text {max }}=20 \mathrm{~m} \mathrm{~s}^{-1}$ at $z=35 \mathrm{~km}$. The jet is Gaussian with a half-width of about $20 \mathrm{~km}$. This configuration can generate a QBO in the absence of planetary wave drag, as shown in Fig. 7a in Part I. With $c_{\max }=60 \mathrm{~m} \mathrm{~s}^{-1}$, the maximum QBO amplitude is about $40 \mathrm{~m} \mathrm{~s}^{-1}$. The high shear regimes in the vicinity of the zonal wind maxima descend to low levels and are eventually dissipated by diffusion near the source level. 
When both types of wave drag are included in the simulation, the profile of equatorial planetary wave drag is affected by the gravity wave-induced high shear regimes. As these high shear regimes descend, $|\bar{u}| \rightarrow$ $c_{\max }$ at the low levels, and since $c_{\max }>c_{\mathrm{PW}},|\bar{u}|$ eventually becomes as large as $c_{\mathrm{PW}}$. This implies that the equatorial planetary waves reach their critical levels, and as implied by (2.1), their drag goes to zero, that is, they are completely absorbed. The gravity waves with absolute value of phase speed greater than $c_{\mathrm{PW}}$, on the other hand, are unaffected by the planetary wave critical levels and their drag still increases exponentially with height within the range of QBO altitudes. Thus, the resulting mean wind oscillation, shown in Fig. 1a, looks similar to the mean wind oscillation that results from gravity wave drag with no planetary wave drag (Fig. 7a in Part I), and the maximum wind amplitude is about the same (Fig. 1b). The oscillation appears to be driven largely by the gravity wave drag, with the contribution from the planetary waves being only at low levels (Fig. 1c). This is in spite of the fact that the total input momentum flux of each sign from each type of wave is the same. The planetary wave contribution has the effect of increasing the rate of descent of the shear zones at the lower levels, compared with that in the case where only gravity wave drag is present (Fig. 7a in Part I). With gravity wave drag only, the shear zone descent rate increases exponentially with height; with the addition of planetary wave drag, there is a more constant descent rate.

The obvious question now is whether it is possible to generate a QBO in which both types of waves contribute to the drag over the whole range of QBO altitudes. To obtain such an oscillation, it is necessary to choose the wave input spectra in such a way that the maximum wind amplitude, as determined by the gravity wave forcing, if it were to act alone, is less than $c_{\mathrm{PW}}$. Since $|\bar{u}| \rightarrow c_{\max }$ as the shear zones descend, the simplest way to ensure this is to choose $c_{\max }<c_{\mathrm{PW}}$. With this requirement in mind, the simulation described above was repeated with $c_{\mathrm{PW}}=50 \mathrm{~m} \mathrm{~s}^{-1}$ instead of $25 \mathrm{~m} \mathrm{~s}^{-1}$ for the planetary waves, and with $c_{\max }=40 \mathrm{~m} \mathrm{~s}^{-1}$ instead of $60 \mathrm{~m} \mathrm{~s}^{-1}$ for the gravity waves. The Newtonian cooling rate was adjusted as well, to maintain the relationship $\mu=1.5 \times k c^{2} / N H$.

With planetary wave drag alone, the mean wind oscillation obtained with $c_{\mathrm{PW}}=50 \mathrm{~m} \mathrm{~s}^{-1}$ is similar to that obtained in Part I, except that the period is longer (approximately $4 \mathrm{yr}$ ). This is because the ratio $N \mu / k c^{2}$, and hence the drag $X^{ \pm}$as given by (2.1), is of the same magnitude as in Fig. 1 of Part I, while the range of wind speeds is now twice as large. Thus, it takes a longer time for the wind to be driven through a QBO cycle. The (a)

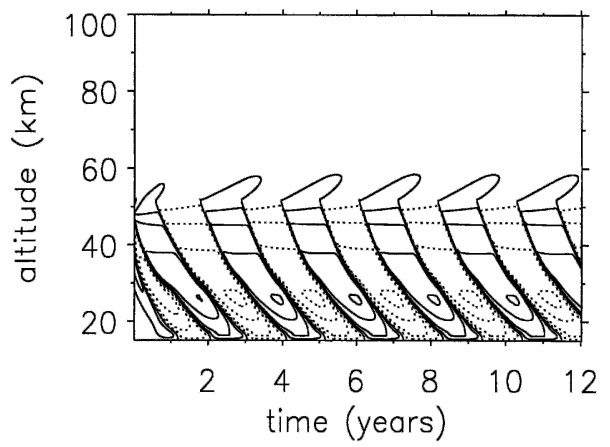

(b)

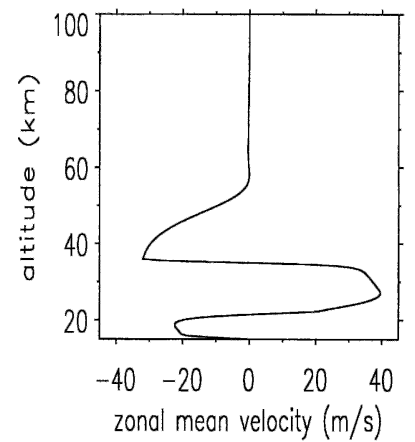

(c)

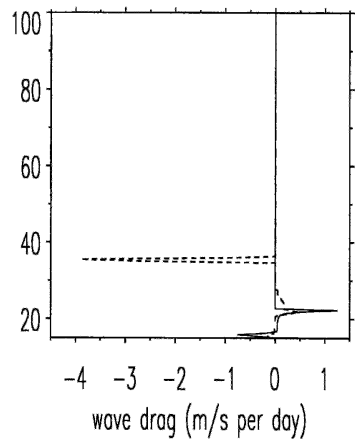

FIG. 1. (a) Time-height plot of the zonal mean wind in a QBO simulation with Kelvin and anti-Kelvin wave drag plus AD99 gravity wave drag with the planetary wave and gravity wave parameters used in Part I: the planetary wave phase speeds are $\pm c_{\mathrm{PW}}= \pm 25 \mathrm{~m} \mathrm{~s}^{-1}$, and the maximum gravity wave phase speed in the input spectrum is $c_{\max }=60 \mathrm{~m} \mathrm{~s}^{-1}$. The solid contours denote westerlies, including the zero-wind line, and the dotted contours denote easterlies. Contour intervals are $10 \mathrm{~m} \mathrm{~s}^{-1}$. (b) Zonal mean wind and (c) planetary wave drag (solid line) and gravity wave drag (dashed line) at the end of the 12-yr run.

time-height plot of the zonal mean velocity for this configuration is shown in Fig. 2 and can be contrasted with Fig. 1 in Part I, where $c_{\mathrm{PW}}=25 \mathrm{~m} \mathrm{~s}^{-1}$. The maximum wind amplitude is now between 20 and $30 \mathrm{~m} \mathrm{~s}^{-1}$.

The time-height plot obtained with gravity waves $\left(c_{\max }=40 \mathrm{~m} \mathrm{~s}^{-1}\right)$ as well as equatorial planetary waves $\left(c_{\mathrm{PW}}=50 \mathrm{~m} \mathrm{~s}^{-1}\right)$ is shown in Fig. 3a. The addition of the gravity wave drag results in a shorter and more realistic period (approximately $2.5 \mathrm{yr}$ ). The maximum wind amplitude is just under $30 \mathrm{~m} \mathrm{~s}^{-1}$ (Fig. 3b). This is a little larger than the maximum wind amplitude obtained when the simulation is carried out with planetary wave drag alone (Fig. 2) or with gravity wave drag alone (not shown here). Figure 3c shows the planetary wave drag and gravity wave drag profiles at the end of the 12-yr run; at low levels, they are of comparable magnitude, but the gravity wave drag is much larger at higher levels because of its exponential increase in mag- 


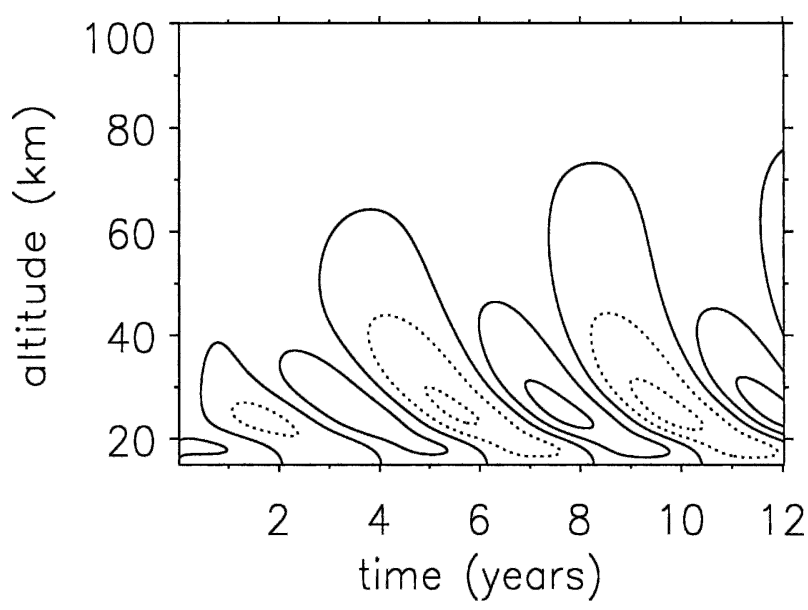

FIG. 2. Time-height plot of the zonal mean wind in a QBO simulation with Kelvin and anti-Kelvin wave drag only. The wave phase speeds are $\pm c_{\mathrm{PW}}= \pm 50 \mathrm{~m} \mathrm{~s}^{-1}$. The Newtonian cooling parameter has been chosen in such a way that the drag, and hence the maximum wind amplitude, decreases exponentially with height. The solid contours denote westerlies, including the zerowind line, and the dotted contours denote easterlies. Contour intervals are $10 \mathrm{~m} \mathrm{~s}^{-1}$.

nitude with height. As before, the total input momentum flux from each type of wave is the same, but the distribution of their drag over the range of QBO altitudes is different. The simulations shown in Figs. 1 and 3 lead us to conclude that the structure of the mean wind oscillation depends on the relative magnitudes of the phase speeds of the two types of waves.

In Fig. 1, the gravity wave drag dominates the planetary wave drag at all but the lowest levels, and so the QBO is largely driven by the gravity wave drag. The opposite situation (in which planetary wave drag dominates) can occur if the planetary wave phase speeds are chosen to be even larger than in Fig. 3, so that the planetary wave-induced wind speed is able to exceed the maximum gravity wave phase speed. This situation can be illustrated by setting $c_{\mathrm{PW}}$ to $100 \mathrm{~m} \mathrm{~s}^{-1}$, with $\mu$ again adjusted to maintain the relationship $\mu=1.5 \times$ $k c^{2} / N H$, while keeping $c_{\max }$ fixed at a value of $40 \mathrm{~m} \mathrm{~s}^{-1}$. First, we investigate the effect of using planetary waves with these large phase speeds without any gravity wave drag. As noted before, in the absence of gravity wave drag, increasing $c_{\mathrm{PW}}$ by a factor of 2 while maintaining the relationship $\mu=1.5 \times k c^{2} / N H$ means that the range of possible wind speeds is twice as large, but the magnitude of the wave drag is unchanged. Thus, in (1.1), the range of possible $\bar{u}$ values is twice as large as before, but $X$ is unchanged; or equivalently, $X$ is a factor of 2 smaller, relative to the other terms in the equation. This means that the diffusion plays a greater role in the evolution of the wind, relative to the effect of the wave (a)

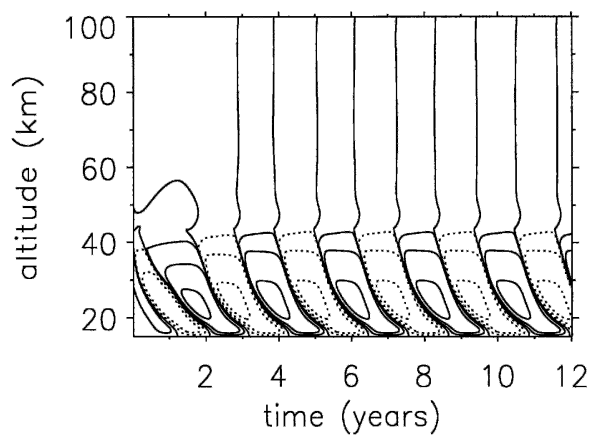

(b)

(c)
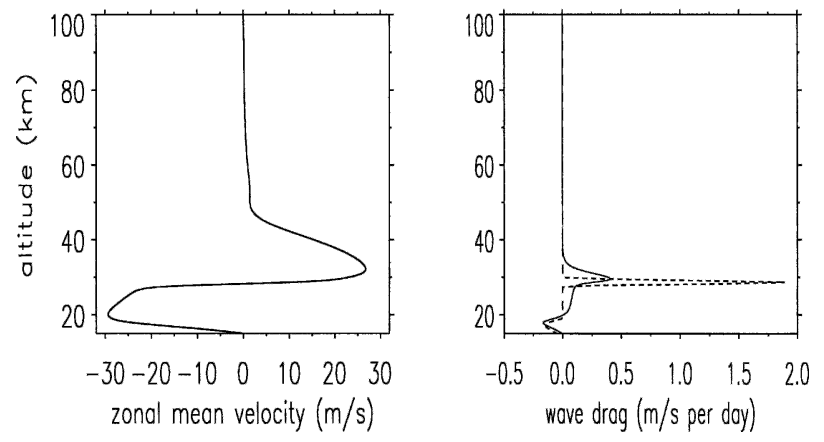

FIG. 3. (a) Time-height plot of the zonal mean wind in a QBO simulation with Kelvin and anti-Kelvin wave drag plus AD99 gravity wave drag. The planetary wave phase speeds are larger and the gravity wave phase speeds are smaller than those in Fig. 1a: the planetary wave phase speeds are $\pm c_{\mathrm{PW}}= \pm 50 \mathrm{~m} \mathrm{~s}^{-1}$ and the maximum gravity wave phase speed in the input spectrum is $c_{\max }=40 \mathrm{~m} \mathrm{~s}^{-1}$. The solid contours denote westerlies, including the zero-wind line, and the dotted contours denote easterlies. Contour intervals are $10 \mathrm{~m} \mathrm{~s}^{-1}$. (b) Zonal mean wind and (c) planetary wave drag (solid line) and gravity wave drag (dashed line) at the end of the 12-yr run.

drag. In fact, with $c_{\mathrm{PW}}$ as large as $100 \mathrm{~m} \mathrm{~s}^{-1}$, the effect of the diffusion dominates the effect of the wave drag. The ratio of the maximum wind amplitude to $c_{\mathrm{PW}}$ depends on the relative magnitude of $X$ to $\nu \partial^{2} \bar{u} / \partial z^{2}{ }^{1}$. Thus, the effect of increasing the effective diffusion is to reduce the strength of the wind relative to $c_{\mathrm{PW}}$. As the wind amplitude decreases, the diffusive term in (1.1) also decreases. However, this term continues to dominate because the total drag $X$ also decreases with de-

\footnotetext{
${ }^{1}$ This is true in general and can be verified quite readily by carrying out experiments with different values of $\nu$ and $F_{0}^{ \pm}$. With $\nu$ reduced and $X$ unchanged, the maximum wind amplitude is larger (i.e., closer to $c_{\mathrm{PW}}$ ). Increasing $\nu$ with $X$ unchanged decreases the strength of the wind. Similarly, if $X$ is reduced (by reducing $F_{0}^{ \pm}$) with $v$ unchanged, the strength of the wind is reduced. With $F_{0}^{ \pm}=0$, there is no wave drag and the wind is soon damped to zero by the diffusion.
} 
creasing $\bar{u}$. The result is a mean wind oscillation with a maximum amplitude of less than $1 \mathrm{~m} \mathrm{~s}^{-1}$. To obtain stronger winds, we must increase the magnitude of the drag as well; to do this, we increase $F_{0}^{ \pm}$also by a factor of 2. Now we have $c_{\mathrm{PW}}=100 \mathrm{~m} \mathrm{~s}^{-1}$ and $F_{0}^{ \pm} / \bar{\rho}_{0}=$ $\pm 0.014 \mathrm{~m}^{2} \mathrm{~s}^{-2}$. The mean wind oscillation obtained with this planetary wave configuration has a maximum amplitude of between 50 and $60 \mathrm{~m} \mathrm{~s}^{-1}$.

Next, we reintroduce the gravity wave drag (with $c_{\max }=40 \mathrm{~m} \mathrm{~s}^{-1}$ ). We now have a situation in which the planetary wave-induced wind speed is greater than the maximum gravity wave phase speed at some levels. The evolution of the mean wind oscillation obtained with this configuration is shown in Fig. $4 \mathrm{a}$, and the wind and drag profiles at the end of the 12-yr run are shown in Figs. 4b,c. The maximum wind speed is just under 60 $\mathrm{m} \mathrm{s}^{-1}$, and the gravity wave drag has no effect on the wind at the levels where the wind speed exceeds 40 $\mathrm{m} \mathrm{s}^{-1}$. Thus, in contrast to Figs. 1 and 3, the planetary wave drag dominates, especially at high levels. This is a more realistic configuration and closer to the situation that actually exists in the terrestrial stratosphere. Finally, we note that adjusting the values of $F_{0}^{ \pm}$(for the planetary waves) or $\varepsilon$ (for the gravity waves) while keeping the wave phase speeds fixed would affect the relative magnitude of the drag from each type of wave, and hence also affect the structure of the oscillation.

The initial mean wind profile used in the simulations shown in Figs. 1-3 is a westerly jet with a maximum of $\bar{u}_{\text {max }}=20 \mathrm{~m} \mathrm{~s}^{-1}$ at $z=35 \mathrm{~km}$. In Part I, it was demonstrated that the ability of the AD99 scheme to generate a QBO depends on the initial mean wind profile. The initial configuration used here gives a realistic QBO with AD99 gravity wave drag alone (Fig. 7 in Part I). However, if the initial shear is too weak (relative to the strength of the vertical diffusion), a situation can result in which the existing shear zones propagate downward but the wind cannot change direction; this means that it is theoretically impossible to have an oscillation between easterlies and westerlies. That is the case if the initial jet used here is replaced by one in which $\bar{u}_{\max }=5 \mathrm{~m} \mathrm{~s}^{-1}$ (see Fig. 6a in Part I).

With planetary wave drag alone, a QBO can develop even if the initial shear is weak (Plumb 1977). With drag from both types of waves, the dependence on the initial shear is more subtle. To illustrate this, we carried out a series of experiments, the results of which are shown in Fig. 5. In Fig. 5a, only planetary wave drag is used; all input parameters are the same as in Fig. $2 \mathrm{a}$ and the only difference is in the initial wind profile, which is a westerly jet with $\bar{u}_{\text {max }}=5 \mathrm{~m} \mathrm{~s}^{-1}$ at $z=35 \mathrm{~km}$. With such a weak initial wind, it takes about $15 \mathrm{yr}$ for the wind to (a)

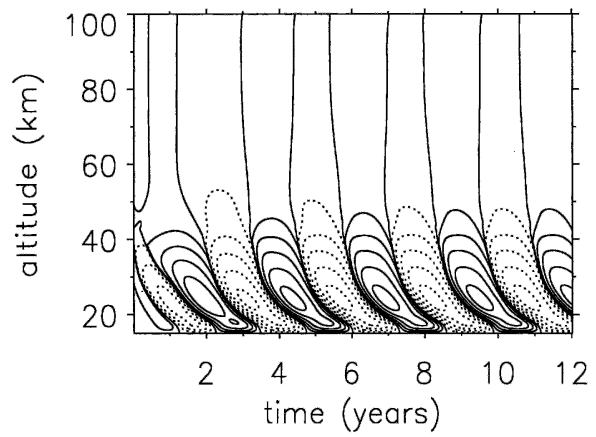

(b)

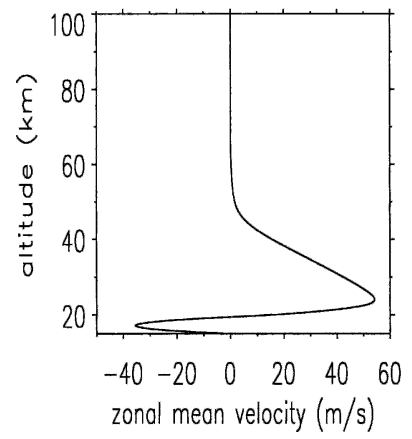

(c)

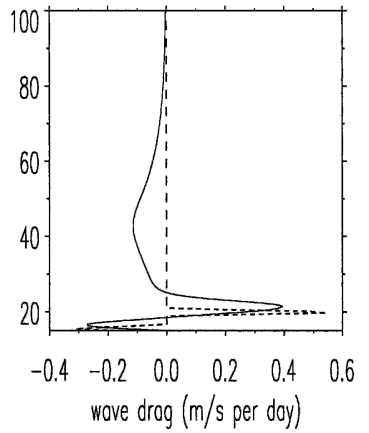

FIG. 4. (a) Time-height plot of the zonal mean wind in a QBO simulation with Kelvin and anti-Kelvin wave drag plus AD99 gravity wave drag. The gravity wave phase speeds are the same as those in Fig. 3: the maximum gravity wave phase speed in the input spectrum is $c_{\max }=40 \mathrm{~m} \mathrm{~s}^{-1}$. The planetary wave phase speeds are twice as large as in Fig. $3\left( \pm c_{\mathrm{PW}}= \pm 100 \mathrm{~m} \mathrm{~s}^{-1}\right)$, and their input momentum fluxes are twice as large as in all the other figures $\left(F_{0}^{ \pm} / \bar{\rho}_{0}= \pm 0.014 \mathrm{~m}^{2} \mathrm{~s}^{-2}\right)$. The solid contours denote westerlies, including the zero-wind line, and the dotted contours denote easterlies. Contour intervals are $10 \mathrm{~m} \mathrm{~s}^{-1}$. (b) Zonal mean wind and (c) planetary wave drag (solid line) and gravity wave drag (dashed line) at the end of the 12-yr run.

build up to its maximum strength of $20-25 \mathrm{~m} \mathrm{~s}^{-1}$, but once it does, the QBO evolves with essentially the same period and vertical structure as in Fig. 2a. In Fig. 5b, only gravity wave drag is used; all gravity wave input parameters are the same as in Fig. 3a and again the only difference is in the initial wind profile, which is the same as in Fig. 5a. The descent of the initial jet can be seen in the first year of the simulation, but above the jet, the wind decreases to zero. A steady state is soon attained, in which the wind is approximately zero at all levels. This is what is predicted by the exact solution given by (4.9) and (4.10) in Part I and shown in Fig. 6a in Part I.

Next we combine the planetary wave drag used in Fig. 5a with the gravity wave drag used in Fig. 5b. The result is shown in Fig. $5 \mathrm{c}$ and is not much different from 
(a)

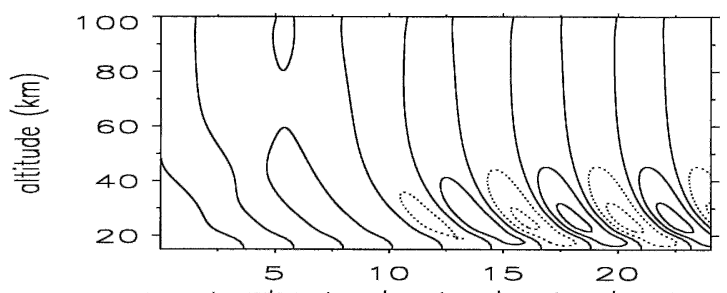

(b)

(c)

(d)
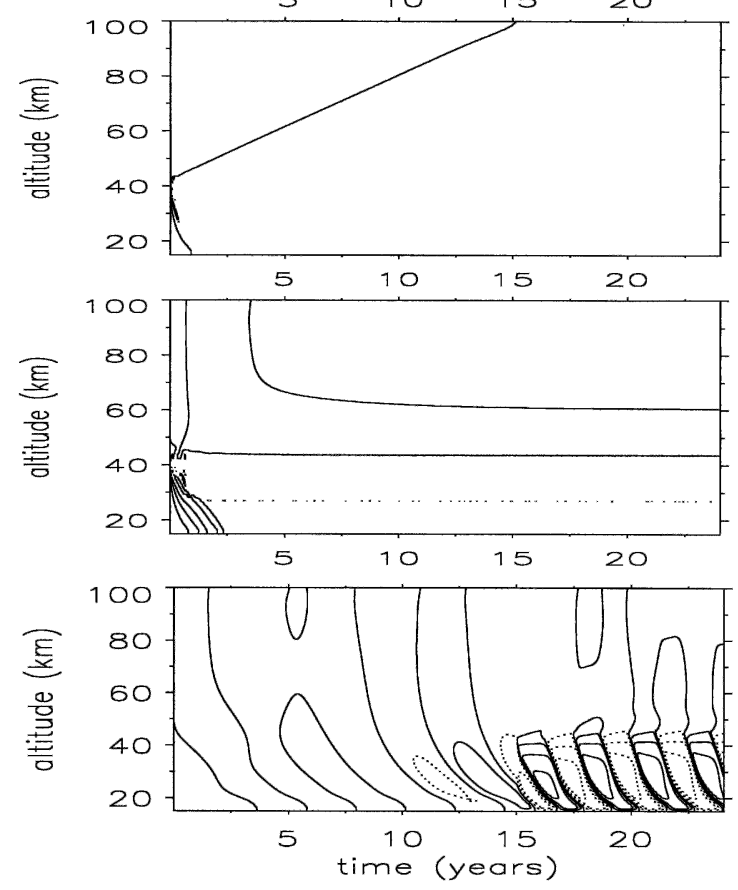

FIG. 5. Time-height plot of the zonal mean wind in a QBO simulation with the planetary wave and gravity wave parameters used in Fig. $3\left(c_{\mathrm{PW}}=50 \mathrm{~m} \mathrm{~s}^{-1}\right.$ and $\left.c_{\max }=40 \mathrm{~m} \mathrm{~s}^{-1}\right)$, but with weak initial mean shear: $u_{\max }=5 \mathrm{~m} \mathrm{~s}^{-1}$, instead of $u_{\max }=20$ $\mathrm{m} \mathrm{s}^{-1}$. The solid contours denote westerlies, including the zerowind line, and the dotted contours denote easterlies. Contour intervals are $10 \mathrm{~m} \mathrm{~s}^{-1}$. (a) Planetary wave drag only, (b) AD99 gravity wave drag only, (c) planetary wave drag plus AD99 gravity wave drag, and (d) planetary wave drag plus AD99 gravity wave drag added after $15 \mathrm{yr}$.

that in Fig. 5b. The jet descends early in the simulation and henceforth a steady state is attained. The presence of the planetary wave drag is barely noticeable. The downward-propagating gravity wave-induced anomaly washes out any possible planetary wave-induced QBO. To understand why this is so, we plotted the drag profiles at the beginning of the simulation. These are shown in Fig. 6; it is clear that the gravity wave drag is several orders of magnitude stronger than the planetary wave drag, and it is not surprising therefore that the effect of the former dominates the latter. As before, the total gravity wave input momentum flux of each sign is of the same order of magnitude as the input momentum flux from the planetary wave of the same sign; in each case, the momentum fluxes from easterly and westerly waves are equal and opposite and thus sum to zero. When $\bar{u}=0$ at all levels, the positive drag (from the westerly waves) and the negative drag (from the easterly waves) cancel out and the total drag is zero. For nonzero $\bar{u}$, the total drag is determined by the extent to which the positive and the negative drag deviate from the values that they have when $\bar{u}$ is zero. Figure $6 \mathrm{~b}$ shows that with this wind profile, the effect of the nonzero $\bar{u}$ on the gravity wave drag is larger than its effect on the planetary wave drag. This is to be expected and results from the difference between the mechanisms for momentum deposition in the two schemes. In the AD99 scheme, waves within a range of phase speeds tend to deposit their momentum over a relatively narrow range of heights so that the resulting drag profile is comprised of narrow large-amplitude spikes. In the HL72 scheme, on the other hand, there is a more gradual deposition of momentum, spread out over a wider range of heights. Narrow spikes in the profile of drag for a given wave occur only in regions where $\bar{u}$ is close to one of the wave phase speeds. Thus, when the wind is weak, as in Fig. 6a, the difference in magnitude between the positive drag and the negative drag (i.e., the total drag) is generally of small amplitude, relative to that in the AD99 scheme, even when the input momentum fluxes are the same in the two schemes.

We can conclude from Fig. 5c that the ability of the AD99 scheme to generate a QBO may depend on the initial wind, even when planetary wave drag is present as well. The determining factor is the relative magnitude of the gravity wave drag to the planetary wave drag. Indeed, repeating this experiment with the gravity wave input momentum flux greatly reduced, it is possible to obtain a QBO. With $\varepsilon$ reduced by a factor of 20 , for example, the planetary wave drag dominates and the wind evolves in a similar manner to that in Fig. 5a and a mean wind oscillation (driven mainly by planetary wave drag) develops after about $15 \mathrm{yr}$ (not shown here).

Finally, we carried out a simulation in which the planetary wave drag was added from the beginning and the wind was allowed to increase in strength over a $15-\mathrm{yr}$ period, and then from $t=15 \mathrm{yr}$ onward, gravity wave drag (with the same input parameters as in Figs. 5b,c) was added. Figure $5 \mathrm{~d}$ shows that the effect of the gravity wave drag is seen almost immediately after it is first introduced. The wind develops a QBO-like oscillation with the same period and structure as that in Fig. 3a. This implies that as long as the shear is strong enough for the gravity wave forcing to take effect at the time it is introduced, a QBO is possible; and once a QBO develops, its form does not depend on the initial wind 
(a)

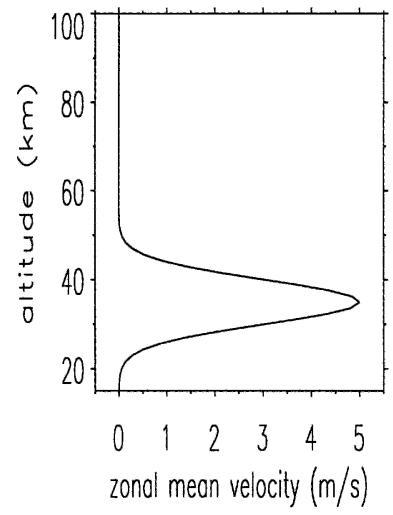

(b)

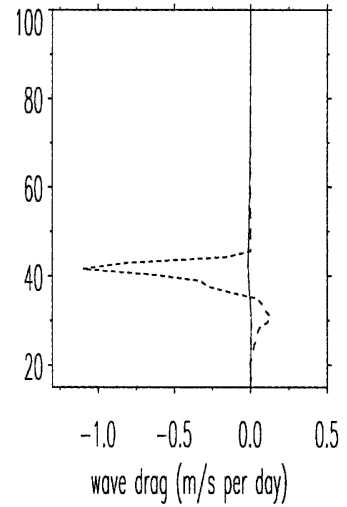

(c)

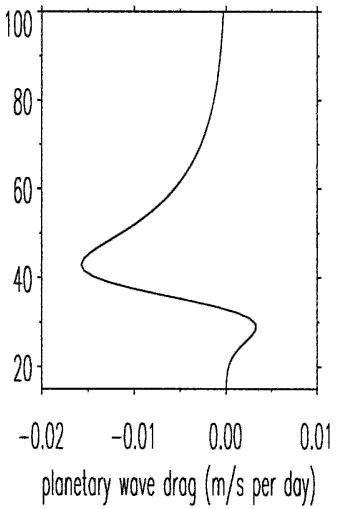

FIG. 6. (a) Initial mean wind used in the simulations shown in Fig. 5. (b) Planetary wave drag (solid line) and gravity wave drag (dashed line) at the beginning of the simulation shown in Fig. 5c. (c) Planetary wave drag from (b), shown on a different scale.

configuration. This is consistent with the conclusions of Part I.

\section{Equatorial planetary wave drag plus Lindzen gravity wave drag}

Lindzen's parameterization (Lindzen 1981; Holton 1982) differs from the AD99 scheme in that each breaking wave deposits its momentum not only at its breaking level but above the breaking level as well, in such a way as to maintain static stability of the wave everywhere above that level. This results in two important differences with respect to the AD99 scheme. The first is that the drag from a single wave can be nonzero all the way up to the upper boundary of the domain under consideration. This means that, in general, any mean wind oscillation generated by this scheme will occur over the whole range of altitudes from the lowest breaking level of the waves up to the upper boundary, even if the source spectrum is truncated at some maximum phase speed. In the AD99 scheme, on the other hand, such a spectrum gives a mean wind oscillation that extends only up to the breaking level of the wave with the largest phase speed in the spectrum. The other important difference is that each wave produces a drag profile that is a piecewise continuous function of height, so a QBO is possible with just two waves (one easterly and one westerly).

We start our investigation by examining the simplest configuration: the case of two waves with equal and opposite phase speeds. This is a suitable parameterization to use to investigate further the constraints on the relative size of the phase speeds of the gravity waves and equatorial planetary waves. In Part I, we showed that the two-wave Lindzen configuration inevitably leads to a QBO-like oscillation (provided that $\bar{u}$ is nonzero initially). The choice of gravity wave input parameters

$$
\begin{aligned}
F_{0}\left(c^{ \pm}\right) / \bar{\rho}_{0} & =F_{0}^{ \pm} / \bar{\rho}_{0}= \pm 7 \times 10^{-3} \mathrm{~m}^{2} \mathrm{~s}^{-2}, \\
c^{ \pm} & = \pm c_{\mathrm{GW}}= \pm 25 \mathrm{~m} \mathrm{~s}^{-1}, \\
k^{ \pm} & =2 \pi /\left(4 \times 10^{7} \mathrm{~m}\right),
\end{aligned}
$$

with intermittency factor $\varepsilon=1$ and vertical diffusivity $\nu=0.4 \mathrm{~m}^{2} \mathrm{~s}^{-1}$ gives a QBO with a period of approximately 2.5 yr (see Fig. 3a in Part I). The wave parameters (3.1) are the same as (2.2), which were used for the planetary waves in section 2 .

We examine three different planetary wave/gravity wave configurations: (a) $c_{\mathrm{PW}}=25 \mathrm{~m} \mathrm{~s}^{-1}, c_{\mathrm{GW}}=50$ $\mathrm{m} \mathrm{s}^{-1}$; (b) $c_{\mathrm{PW}}=25 \mathrm{~m} \mathrm{~s}^{-1}, c_{\mathrm{GW}}=25 \mathrm{~m} \mathrm{~s}^{-1}$; and (c) $c_{\mathrm{PW}}=50 \mathrm{~m} \mathrm{~s}^{-1}, c_{\mathrm{GW}}=25 \mathrm{~m} \mathrm{~s}^{-1}$. The results are shown in Figs. 7a,b,c respectively. With the configuration (a), a situation similar to that shown in Fig. 1 occurs; the large phase speed gravity waves create strong winds (with speeds of up to $46 \mathrm{~m} \mathrm{~s}^{-1}$ ), and the small phase speed planetary waves are absorbed at low levels. The result is a mean-wind oscillation that is driven almost completely by gravity waves alone; Fig. 7a is almost identical to what is obtained without the planetary waves (not shown). With configuration (b), the maximum wind amplitude is about $23 \mathrm{~m} \mathrm{~s}^{-1}$. The period of the mean-wind oscillation is shorter than in (a), because it takes a shorter time for the wind to be driven through a QBO cycle. The planetary wave contribution is seen in the lower part of the computational domain (below $z=50 \mathrm{~km}$ ), but the gravity wave drag still dominates, 
(a)

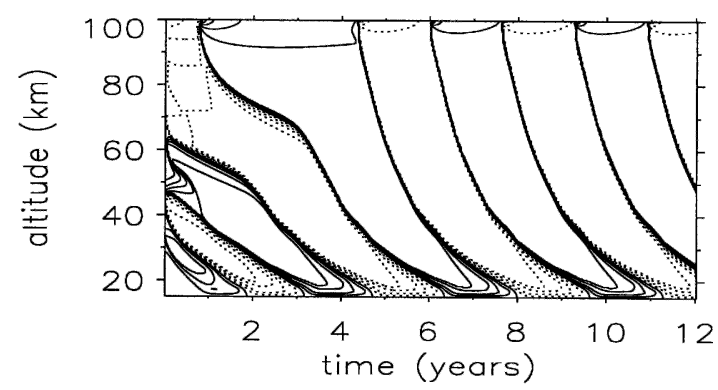

(b)

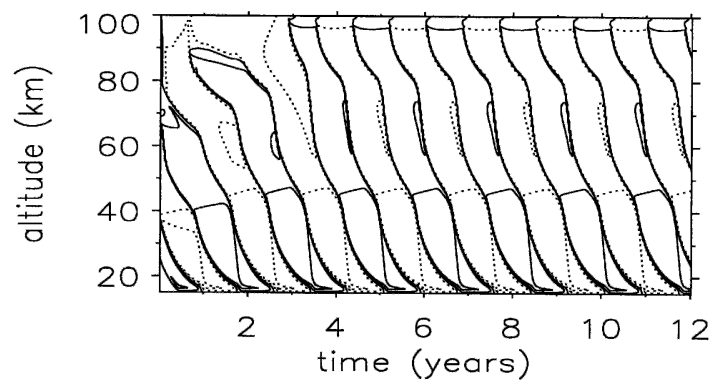

(c)

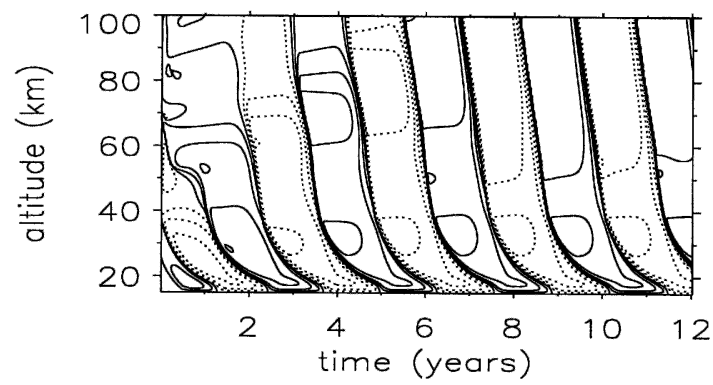

FIG. 7. Time-height plot of the zonal mean wind in a QBO simulation with Kelvin and anti-Kelvin wave drag plus Lindzen gravity wave drag (two waves with equal and opposite phase speeds). The solid contours denote westerlies, including the zerowind line, and the dotted contours denote easterlies. Contour intervals are $10 \mathrm{~m} \mathrm{~s}^{-1}$. (a) The planetary wave phase speeds are $\pm c_{\mathrm{PW}}= \pm 25 \mathrm{~m} \mathrm{~s}^{-1}$ and the gravity wave phase speeds are $\pm c_{\mathrm{GW}}$ $= \pm 50 \mathrm{~m} \mathrm{~s}^{-1}$. (b) Same as in (a), but with $c_{\mathrm{PW}}=25 \mathrm{~m} \mathrm{~s}^{-1}$ and $c_{\mathrm{GW}}$ $=25 \mathrm{~m} \mathrm{~s}^{-1}$. (c) Same as in (a), but with $c_{\mathrm{PW}}=50 \mathrm{~m} \mathrm{~s}^{-1}$ and $c_{\mathrm{GW}}$ $=25 \mathrm{~m} \mathrm{~s}^{-1}$.

especially above this level. This is because, although the planetary waves do not actually have critical levels, their drag becomes small as the wind is driven close to its maximum strength. With configuration (c), on the other hand, the planetary wave effects are larger and can be seen even at higher altitudes; in fact the planetary wave drag is at times nonzero near the top of the computational domain. The maximum wind speeds in this case are about $45 \mathrm{~m} \mathrm{~s}^{-1}$, but in contrast to (a), these high-speed winds are driven by planetary waves, rather than gravity waves. Of the three configurations, the last is the most realistic as a model for the terrestrial QBO, in the sense that the $\mathrm{QBO}$ is driven primarily by equa- torial planetary wave drag and is enhanced by gravity wave drag.

The simulations described above made use of an idealized model of the equatorial planetary waves in which the effect of the earth's rotation on the easterly wave was ignored. An obvious question is whether our conclusions can be extended to a more realistic planetary wave configuration. To answer this question, we carried out some QBO simulations using gravity wave drag in combination with drag from a westerly Kelvin wave and a true easterly mixed Rossby-gravity wave in which the effect of rotation was represented. The results of these simulations (not shown here) were not much different qualitatively from those obtained with an easterly antiKelvin wave, and this suggests that our conclusions are indeed robust to differences in the planetary wave configuration.

\section{Discussion}

We have examined the effect of combining drag from gravity waves and equatorial planetary waves in our simple one-dimensional model of the QBO. We found that the ability of the model to generate a realistic QBO depends on a number of factors: the relative magnitude of the phase speeds of the two types of waves, the relative magnitude of their input momentum fluxes, and the strength of the initial wind speeds.

If the gravity wave phase speeds are too large relative to those of the planetary waves, the gravity waves can induce regions of high shear in which the planetary waves undergo critical level absorption. The result is that above the highest planetary wave critical level, the mean wind oscillation is driven only by gravity waves. However, provided the gravity wave phase speeds are small enough relative to those of the planetary waves, it is possible to obtain a QBO to which both types of waves contribute. This is a more realistic representation of the situation that exists in the terrestrial stratosphere. Under these conditions, the gravity wave drag acts to give a stronger QBO with a shorter period than that which would be obtained with planetary wave drag alone. The structure of the QBO depends on the relative magnitude of the input momentum fluxes of the two types of waves. These conclusions suggest that if the drag from equatorial planetary waves is insufficient to drive a realistic QBO (e.g., in models that include tropical upwelling), gravity wave drag can be added to compensate.

We have investigated the effect of the initial wind profile on the ability of the wave drag schemes to generate a QBO. From Part I, we know that the ability of the AD99 gravity wave scheme to generate a QBO 
depends on the initial wind profile; if the initial shear is too weak (relative to the strength of the vertical diffusion), it may be impossible to obtain a mean-wind oscillation between positive and negative values. However, the ability of the HL72 planetary wave scheme and the Lindzen gravity wave scheme to generate a QBO is less dependent on the initial wind profile.

We have shown here that, somewhat surprisingly, the constraints on the initial wind profile in the AD99 scheme apply even when the AD99 gravity wave drag is combined with planetary wave drag. We examined a configuration in which the initial wind was so weak that it was impossible to obtain a QBO with AD99 gravity wave drag alone. We saw that even with the addition of HL72 planetary wave drag, there was no guarantee that a QBO would result. The gravity wave drag was seen to be several orders of magnitude larger than the planetary wave drag, when their input momentum fluxes were the same, and thus the gravity wave drag dominated. To obtain a QBO in such a case, one would need to decrease the gravity wave input momentum flux to reduce the gravity wave drag to the order of magnitude of the planetary wave drag. Alternatively, one could add the planetary wave drag to the model first and allow enough time for the planetary wave-induced winds to grow to large amplitude before adding the gravity wave drag. A simulation of this sort was carried out and the resulting QBO was almost identical to that which would be obtained if the initial wind had been strong enough for the gravity wave drag to drive a QBO.

The one-dimensional model used here and in Part I does not include the effect of upwelling, and this is an obvious deficiency, considering the profound effect that upwelling has on the QBO. Dunkerton (1997) noted that, in general, the rate of upwelling may be comparable, but opposite in sign, to the rate of descent of QBO shear zones. Also, there is reason to believe that the semiannual oscillation (SAO) at the stratopause plays a role in initiating the downward propagating phases of the QBO, in the sense that westerly QBO phases are connected to the descending westerly regimes of the SAO (Dunkerton 1990). In Part I, we discussed the question of whether there is downward propagation of influence in the QBOs simulated by the different parameterization schemes. However, since our one-dimensional model does not simulate the SAO, any investigation into synchronization of the $\mathrm{SAO}$ with the QBO is beyond the scope of the study described here and in Part I. The next step in our investigation involves adding parameterized wave drag to a two-di- mensional balance model that simulates the seasonal cycle, including the stratopause SAO and upwelling in the Tropics (Semeniuk and Shepherd 2001). The ultimate goal is to extend the constraints derived using these simple one- and two-dimensional models to provide some guidance for the use of gravity wave drag parameterization schemes in GCMs.

Acknowledgments. This research was supported by the Natural Sciences and Engineering Research Council of Canada, in part through the Modelling of Global Chemistry for Climate (GCC) project. The authors are grateful to Drs. C. McLandress and M. J. Alexander for helpful discussions, and to Dr. T. J. Dunkerton and an anonymous referee for constructive criticism of the manuscript.

\section{REFERENCES}

Alexander, M. J., and T. J. Dunkerton, 1999: A spectral parameterization of mean-flow forcing due to breaking gravity waves. J. Atmos. Sci., 56, 4167-4182.

Campbell, L. J., and T. G. Shepherd, 2005: Constraints on wave drag parameterization schemes for simulating the quasibiennial oscillation. Part I: Gravity wave forcing. J. Atmos. Sci., 62, 4178-4195.

Dunkerton, T. J., 1990: Annual variation of deseasonalized mean flow acceleration in the equatorial lower stratosphere. J. Meteor. Soc. Japan, 68, 499-508.

- 1997: The role of gravity waves in the quasi-biennial oscillation. J. Geophys. Res., 102, 26 053-26076.

Giorgetta, M. A., E. Manzini, and E. Roeckner, 2002: Forcing of the quasi-biennial oscillation from a broad spectrum of atmospheric waves. Geophys. Res. Lett., 29, 1245, doi: 10.1029/2002GL014756.

Holton, J. R., 1982: The role of gravity wave induced drag and diffusion in the momentum budget of the mesosphere. J. Atmos. Sci., 39, 791-799.

_ , and R. S. Lindzen, 1972: An updated theory for the quasibiennial cycle of the tropical stratosphere. J. Atmos. Sci., 29, 1076-1080.

Horinouchi, T., and Coauthors, 2003: Tropical cumulus convection and upward propagating waves in middle atmospheric GCMs. J. Atmos. Sci., 60, 2765-2782.

Lindzen, R. S., 1981: Turbulence and stress owing to gravity wave and tidal breakdown. J. Geophys. Res., 86, 9707-9714.

McLandress, C., 2002: Interannual variations of the diurnal tide in the mesosphere induced by a zonal-mean wind oscillation in the tropics. Geophys. Res. Lett., 29, 1305, doi:10.1029/ 2001GL014551.

Plumb, R. A., 1977: The interaction of two internal waves with the mean flow: Implications for the theory of the quasi-biennial oscillation. J. Atmos. Sci., 34, 1847-1858.

Scaife, A. A., N. Butchart, C. D. Warner, D. Stainforth, W. Norton, and J. Austin, 2000: Realistic quasi-biennial oscillation in a simulation of the global climate. Geophys. Res. Lett., 27, 3481-3484.

Semeniuk, K., and T. G. Shepherd, 2001: Mechanisms for tropical upwelling in the stratosphere. J. Atmos. Sci., 58, 3097-3115. 